\title{
Cheap Control Tracking Performance for Non-Right-Invertible Systems ${ }^{1}$
}

\author{
A.R. Woodyatt ${ }^{2}$, M.M. Seron ${ }^{3}$, J.S. Freudenberg ${ }^{4}$, R.H. Middleton ${ }^{5}$ \\ Department of Electrical and Computer Engineering \\ The University of Newcastle, NSW 2308 Australia
}

\begin{abstract}
A fundamental limitation exists in the achievable tracking performance of non-right-invertible systems. This limitation manifests itself in the cheap control tracking cost, which we show to be a function of the plant non-minimum phase zeros and of the variation with frequency of the plant direction. The cheap control tracking cost is further connected with an integral relation that constrains the performance of any stable closedloop system where the plant has a single input and two outputs.
\end{abstract}

\section{Introduction}

When designing control systems, there is a tradeoff between small amplitude inputs and fast transient response of the closed loop system. Large amplitude input signals may be required to achieve small deviations of the system from the desired trajectory. This tradeoff becomes evident in the cheap control problem, which is concerned with the limiting behaviour of the linear quadratic regulator as the cost in control tends to zero.

The asymptotic value of the cheap control cost has been studied by many authors $[7,12,8,9,3,11]$. In particular, Kwakernaak and Sivan [7] showed that, if the system is minimum phase and right-invertible, then the cheap control cost will become arbitrarily close to zero as the control cost becomes arbitrarily small. On the other hand, if the system is either non-minimum phase or non-right-invertible (or both), then the cheap control cost will be nonzero. Kwakernaak and Sivan also showed that if the plant is non-minimum phase and square then as the control cost approaches zero some of the closed loop poles will tend to positions in the left

\footnotetext{
${ }^{1}$ Work supported by the Australian Research Council, U. S. National Science Foundation, and Ford Motor Co.

2 woody@ee.newcastle.edu.au

${ }^{3}$ serondee.newcastle.edu.au

${ }^{4}$ J.S. Freudenberg is with the Department of Electrical Engineering and Computer Science, University of Michigan, Ann Arbor, MI 48109-2122 USA, jfroeecs. umich.edu

5 rick@ee.newcastle .edu . au 0-7803-4394-8/98 \$10.00 (C) 1998 IEEE
}

half plane determined by the non-minimum phase zeros of the plant. For non-right-invertible systems, similar results apply except that some of the closed loop pole positions arise because the plant is non-square $[2,5]$.

Here we are interested in the value of the cheap control tracking cost, where the performance variable in the quadratic cost functional is the deviation of the system output from a constant setpoint. For non-minimum phase and right-invertible systems, Qiu and Davison expressed the cheap control tracking cost as a function of the plant non-minimum phase zeros [8]. For nonright-invertible systems, we show that, in addition to being a function of the plant non-minimum phase zeros, the cheap control tracking cost is increased by a term which is a function of the variation with frequency of the plant direction.

The remainder of this paper is organised as follows. Section 2 contains definitions and derives some results required for the development of this paper. Section 3 gives a formula for the cheap control tracking cost for non-right-invertible systems. Section 4 provides a connection, for single input two output systems, between the cheap control result given in Section 3 and integral relations that have previously been developed. Finally, some examples and conclusions are given.

\subsection{Notation and Definitions}

A transfer matrix is a matrix whose entries are rational functions of the complex variable $s$. The normal rank of a transfer matrix $P$ is the maximal possible rank of $P(s)$ for at least one $s \in \mathbf{C}$. A non-right-invertible transfer matrix has full normal rank if its normal rank is equal to the number of its columns.

Let the open and closed right and left halves of the complex plane be denoted by ORHP, CRHP, OLHP, and CLHP, respectively. We use the acronyms SITO for "single input two output"; and FIMO for "few inputs many outputs". A transfer matrix with more rows than columns will be referred to as a FIMO transfer matrix for brevity. A FIMO transfer matrix with full row rank is non-right invertible. 
The zeros (poles) of a transfer matrix are defined to be the roots of the numerator (denominator) polynomials of the nonzero entries of its Smith-McMillan form. A transfer matrix is stable if all its poles are in the OLHP; minimum phase if all its zeros are in the CLHP, otherwise it is non-minimum phase.

A transfer function $P_{i}$ is said to be inner if $P_{i}$ is stable and $P_{i}^{\mathrm{T}}(-s) P_{i}(s)=I, \quad \forall s \in \mathrm{C}$. A transfer function $P_{o}$ is said to be outer if it has full row rank in the ORHP.

\section{Preliminaries}

\subsection{Plant Direction and DC coordinates}

For the purposes of this paper, the plant is a strictly proper $q \times p$ FIMO transfer matrix $P$ of full normal rank. An important concept associated with a $q \times p$ FIMO transfer matrix $P$ is its direction. If $P$ has normal rank equal to $p$, then its direction at $s \in \mathbf{C}$ may be defined as the $p$-dimensional subspace of $\mathbf{C}^{q}$ spanned by $P(s)$, away from its zeros and poles.

The precise definition of direction uses a particular transfer matrix factorization. For example, in the SITO case, we define the direction of

$$
P(s)=\left[\begin{array}{c}
\frac{s+1}{(2 s+1)(0.3 s+1)} \\
\frac{s}{(0.3 s+1)^{2}}
\end{array}\right]
$$

as the range - at each $s \in \mathbf{C}$ - of the polynomial matrix

$$
N(s)=\left[\begin{array}{c}
(s+1)(0.3 s+1) \\
s(2 s+1)
\end{array}\right]
$$

obtained from the factorization

$$
P(s)=N(s) \frac{1}{(2 s+1)(0.3 s+1)^{2}} .
$$

Observe that $N$ in (2.1) spans a one-dimensional subspace of $\mathbf{C}^{2}$ for all $s \in \mathbf{C}$. A factorization of the form (2.2) can always be obtained in the general FIMO case as shown below.

Lemma 2.1 A $q \times p$ transfer matrix $P$ of normal rank equal to $p$ can aluays be factored as

$$
P(s)=N(s) K(s) \text {, }
$$

where $N$ is a $q \times p$ polynomial matrix, and $K$ is a $p \times p$ transfer matrix. Moreover, $N$ has the following properties:

(a). $\operatorname{rank} N(s)=p$ for all $s \in \mathbf{C}$; (b). $N$ is invariant under feedback of the states of any controllable and observable realization of $P$.

Proof: See Woodyatt et. al. [16].

We use the factorization of $P$ given in Lemma 2.1 to define its direction.

Definition 2.2 (Transfer Matrix Direction) Let $P(s)=N(s) K(s)$ be as in Lemma 2.1. Then its direction at each $s \in \mathbf{C}$ is the range of the matrix $N(s)$. If $N$ is a constant matrix, we say that the transfer function direction is constant with frequency.

o

The direction of the transfer matrix $P$ is referred to as plant direction.

Many properties of a feedback system at a given frequency $s=j \omega$ depend only upon the directions of the plant and controller at that frequency [4]. Moreover, the way in which plant direction changes with frequency imposes closed-loop performance limitations. These limitations were previously expressed [14] as integral constraints on closed-loop transfer functions, and are further investigated here in the cheap control tracking cost.

To describe how the plant direction varies with frequency it is convenient to use a special coordinate basis for the output space. The idea is that, in the new coordinates, the direction at DC $(s=0)$ of a $q \times p$ FIMO plant can be "normalized" to be the range space of the matrix $\left[\begin{array}{ll}I_{p} & 0\end{array}\right]^{\mathrm{T}}$, where $I_{p}$ is the $p \times p$ identity matrix.

Definition 2.3 (DC Coordinates) Let $P=N K$ be as in Lemma 2.1 and choose a constant orthogonal matrix $M \in \mathbf{R}^{q \times q}$ such that

$$
M^{T} N(0)=\left[\begin{array}{l}
V \\
0
\end{array}\right]
$$

where $V$ is a square constant matrix with full rank.

Let $\tilde{N}(s)=M^{T} N(s)$. Then the plant in $D C$ coordinates $P$ is defined as

$$
\tilde{P}(s)=\bar{N}(s) K(s) .
$$

Note that $\tilde{P}(s)=M^{\mathrm{T}} P(s)$ and its direction at $s=0$ is the range of $\tilde{N}(0)=\left[\begin{array}{ll}V^{\mathbf{T}} & 0\end{array}\right]^{\mathbf{T}}$.

\subsection{Inner-Outer Factorization in DC Coordi- nates}

We define

$$
\Delta(s)=N^{\mathbf{T}}(-s) N(s),
$$


or, equivalently, $\Delta(s)=\tilde{N}^{\mathrm{T}}(-s) \tilde{N}(s)$.

Note that $\Delta$ has no zeros on the imaginary axis because $\Delta(j \omega)=N^{\mathrm{H}}(j \omega) N(j \omega)$ and hence rank $\Delta(j \omega)=$ $\operatorname{rank} N(j \omega)=p$ (cf. Lemma 2.1). We can then perform a spectral factorization of $\Delta$, namely, find a "minimum phase" polynomial matrix (that is, with all its zeros in the OLHP) $\Delta_{M P}$ such that

$$
\Delta(s)=\Delta_{M P}^{\mathrm{T}}(-s) \Delta_{M P}(s) .
$$

With $\Delta_{M P}$ so defined, and $K$ as in (2.4), let an innerouter factorization of $\Delta_{M P} K$ be

$$
\Delta_{M P}(s) K(s)=\left[\Delta_{M P}(s) K(s)\right]_{i}\left[\Delta_{M P}(s) K(s)\right]_{0},
$$

where $\left[\Delta_{M P} K\right]_{i}$ is inner, and its zeros are the nonminimum phase zeros of $K$, and its poles are the mirror image (with respect to the imaginary axis) of the nonminimum phase zeros of $K$.

With the above definitions, an inner-outer factorization of $\tilde{P}$ is obtained as follows.

Lemma 2.4 An inner-outer factorization of $\tilde{P}=\tilde{N} K$ is

$$
\tilde{P}(s)=\tilde{P}_{i}(s) \tilde{P}_{o}(s)
$$

where

$$
\begin{aligned}
\tilde{P}_{i}(s) & =\tilde{N}(s) \Delta_{M P}^{-1}(s)\left[\Delta_{M P}(s) K(s)\right]_{i} U \\
\tilde{P}_{o}(s) & =U^{T}\left[\Delta_{M P}(s) K(s)\right]_{i}^{-1} \Delta_{M P}(s) K(s)
\end{aligned}
$$

with $U \in \mathbf{R}^{p \times p}$ any orthogonal matrix.

Proof: Clearly, multiplying (2.7) and (2.8) yields $\tilde{P}$. As all the zeros of $\Delta_{M P}$ are minimum phase and $\left[\triangle_{M P} K\right]_{i}$ is stable, if follows that $\tilde{P}_{i}$ is stable. Furthermore, easy calculations show that $\tilde{P}_{i}^{\mathrm{T}}(-s) \tilde{P}_{i}(s)=I$, and so $\tilde{P}_{i}(s)$ is inner. Also, by construction, $\tilde{P}_{0}$ is minimum phase and right invertible. Hence $\tilde{P}_{i} \tilde{P}_{o}$ is an inner-outer factorization of $\tilde{P}$.

\section{Cheap Control Tracking Problem}

The fact that a FIMO system

$$
y=P u
$$

has fewer inputs than outputs limits the set of setpoints that are admissible, that is, that the output $y$ can track with zero steady-state error. If $P=N K$ is a factorization as in Lemma. 2.1, and $K$ has no zeros at $s=0$, then the set of admissible setpoints for the output $y$ is the set of all $r \in \mathbf{R}^{q \times 1}$ that belong to the range of $N(0)$, that is, they are aligned with the plant direction at DC. We restrict our attention to plants that have no zeros at $s=0$ and to the set of admissible setpoints.

\section{Assumption 3.1}

(i). The $q \times p$ FIMO plant $P=N K$ has no zeros at $s=0$ (equivalently, $K$ has no zeros at $s=0$ ).

(ii). The setpoint $r \in \mathbf{R}^{q \times 1}$ is admissible, that is, it belongs to the range of $N(0)$.

For each admissible setpoint there exists an input $u$ that makes the output $y$ track it asymptotically. Specifically, if $\dot{x}=A x+B u, y=C x$ is a stabilizable and detectable realization of $P(s)$ and $r$ is an admissible setpoint, then we can find [16] $F_{1} \in \mathbf{R}^{p \times p}$ and $r_{1} \in \mathbf{R}^{p \times 1}$ such that the controller

$$
u=F x+F_{1} r_{1},
$$

where $A+B F$ is Hurwitz, achieves asymptotic setpoint tracking $y(t) \rightarrow r$ as $t \rightarrow \infty$. Denoting the tracking error by $e=y-r$, and the transient part of the input by $u_{e}=u-\bar{u}$, where $\bar{u}$ is the steady-state value of $u$, and assuming zero initial conditions for the state $x$, we evaluate tracking performance through the cost functional

$$
J\left(u_{e}, r, \varepsilon\right)=\int_{0}^{\infty} e^{\mathrm{T}}(t) e(t)+\varepsilon^{2} u_{e}^{\mathrm{T}}(t) u_{e}(t) d t .
$$

Let the minimum value of (3.1), obtained for the optimal control $u_{e}=u_{e}^{o p t}$, be

$$
J^{o p t}(r, \varepsilon)=J\left(u_{e}^{o p t}, r, \varepsilon\right) .
$$

Then the best tracking performance is obtained in the limit as $\varepsilon \rightarrow 0$, that is,

$$
\lim _{\varepsilon \rightarrow 0} J^{o p t}(r, \varepsilon) .
$$

The limit (3.2) is the cheap control tracking cost, so called because the cost of control in (3.1) becomes negligible as $\varepsilon \rightarrow 0$. For square systems $(q=p)$, Qiu and Davison [8] showed that (3.2) is a function of the plant non-minimum phase zeros. Here we will show that for FIMO systems $(q>p)$ there exists an additional nonnegative cost due to the variation with frequency of the plant direction.

Note that, from (2.3), if $r$ is an admissible setpoint for the plant in original coordinates, then the corresponding admissible setpoint for the plant in DC coordinates, $M^{\mathrm{T}} r$, has the form

$$
M^{\mathrm{T}} r=\left[\begin{array}{c}
r_{1} \\
0
\end{array}\right],
$$

where $r_{1} \in \mathbf{R}^{p \times 1}$. 
Consider the $q \times p$ polynomial matrix $\tilde{N}=M^{\mathrm{T}} N$ introduced in Definition 2.3. Partition it as

$$
\tilde{N}(s)=\left[\begin{array}{l}
\tilde{N}_{1}(s) \\
\tilde{N}_{2}(s)
\end{array}\right]
$$

such that $\tilde{N}_{1}$ is square. We define the sets:

$\left\{\delta_{i}: i=1, \ldots, N_{\delta}\right\}$ of ORHP zeros of $\Delta$ defined in (2.5); $\left\{\rho_{i}: i=1, \ldots, N_{\rho}\right\}$ of ORHP zeros of $\tilde{N}_{1}$; $\left\{-\lambda_{i}: i=1, \ldots, N_{\lambda}\right\}$ of OLHP zeros of $\tilde{N}_{1}$; $\left\{\alpha_{i}: i=1, \ldots N_{\alpha}\right\}$ of ORHP zeros of $K$.

Then the cheap control tracking cost (3.2) has the following form.

Theorem 3.2 Under Assumption 3.1 the cheap control tracking cost (3.2) is given by

$$
\lim _{\varepsilon \rightarrow 0} J^{o p t}(r, \varepsilon)=r_{1}^{T} H r_{1}
$$

where $r_{1}$ is defined in (3.3) and where

$$
\begin{aligned}
& \text { trace } H=2\left[\sum_{i=1}^{N_{\alpha}} \frac{1}{\alpha_{i}}+\sum_{i=1}^{N_{\rho}} \frac{1}{\rho_{i}}\right]+\operatorname{Var}(P), \\
& \operatorname{Var}(P)=\sum_{i=1}^{N_{\delta}} \frac{1}{\delta_{i}}-\sum_{i=1}^{N_{\lambda}} \frac{1}{\lambda_{i}}-\sum_{i=1}^{N_{\rho}} \frac{1}{\rho_{i}} .
\end{aligned}
$$

Proof: See Woodyatt et. al. [16].

Theorem 3.2 shows that the cheap control tracking cost for FIMO systems is $r_{1}^{\mathrm{T}} H r_{1}$, and (3.6) gives an expression for trace $H$ as an explicit function of the zeros defined in (3.5). Theorem 3.2 shows that when the plant is non-right-invertible, the cheap control tracking cost is not only a function of the non-minimum phase zeros of the system - given by the term between square brackets in (3.6) - but there is also an additional term $\operatorname{Var}(P)$ which, as we show next, is due to the change in the direction of the plant.

Lemma 3.3 For a plant $\tilde{P}$ in DC coordinates, partition the plant as

$$
\tilde{P}(s)=\left[\begin{array}{l}
\tilde{P}_{1}(s) \\
\tilde{P}_{2}(s)
\end{array}\right]
$$

such that $\tilde{P}_{1}$ is square. Then

$\frac{1}{\pi} \int_{0}^{\infty} \log \left|\operatorname{det}\left(I+\tilde{P}_{\text {rat }}^{T}(-j \omega) \tilde{P}_{\text {rat }}(j \omega)\right)\right| \frac{d \omega}{\omega^{2}}=\operatorname{Var}(P)$,

where $\tilde{P}_{r a t}(s)=\tilde{P}_{2}(s) \tilde{P}_{1}^{-1}(s)$.
Proof: See Woodyatt et. al. [16].

If the plant direction varies significantly with frequency, in the sense that the integral in (3.9) is large, then trace $H$ in (3.6) will be large. Therefore, variations of the plant direction with frequency imply a fundamental limitation in the tracking ability of FIMO linear systems using full state feedback. This limitation has no analog in square multivariable systems.

When the plant direction is constant with frequency we recover the result for the square multivariable case.

Corollary 3.4 Under Assumption 3.1, if the plant direction is constant with frequency, the cheap control tracking cost (3.2) is given by

$$
\lim _{\varepsilon \rightarrow 0} J^{o p t}(r, \varepsilon)=r_{1}^{T} H r_{1}
$$

where $r_{1}$ is defined in (3.3) and where

$$
\operatorname{trace} H=2 \sum_{i=1}^{N_{\text {v }}} \frac{1}{\alpha_{i}} \text {. }
$$

Proof: If the plant direction is constant with frequency, then by definition the matrix $N$ is constant. Therefore the sets $\left\{\delta_{i}: i=1, \ldots, N_{\delta}\right\},\left\{\rho_{i}: i=\right.$ $\left.1, \ldots, N_{\rho}\right\}$ and $\left\{-\lambda_{i}: i=1, \ldots, N_{\lambda}\right\}$ are all empty, and the result follows.

\section{Connection with an Integral Relation for Single Input Two Output Systems}

We have shown that there is a simple expression for the cheap control tracking cost for FIMO systems. We now provide a link, in the SITO case, between the cheap control tracking cost and an integral relation on the output complementary sensitivity function.

Consider a linear time-invariant SITO plant, controlled by a linear time-invariant controller. Let the plant and controller be represented by rational and proper transfer functions $P(s)=\left[\begin{array}{ll}p_{1}(s) & p_{2}(s)\end{array}\right]^{\mathrm{T}}$ and $C(s)$. Define the input and output open loop transfer functions, as $L_{r}(s) \triangleq C(s) P(s)$ and $L_{O}(s) \triangleq P(s) C(s)$ respectively; and the input and output complementary sensitivity functions as as $T_{I}(s) \triangleq L_{I}(s)\left(1+L_{I}(s)\right)^{-1}$ and $T_{O}(s) \triangleq L_{O}(s)\left(I+L_{O}(s)\right)^{-1}$ respectively. Furthermore, denote by $T_{O \bullet 1}$ and $T_{O 11}$ the first column of $T_{O}$, and the element in the first row and first column of $T_{O}$ respectively. Consistent with previous notation, denote the output complementary sensitivity function in DC coordinates by $\tilde{T}_{O}$, where $\tilde{T}_{O} \triangleq \tilde{P} \tilde{C}(I+\tilde{P} \tilde{C})^{-1}$. The norm of the output complementary sensitivity function 
is invariant under transformation to $\mathrm{DC}$ coordinates, that is $\left\|T_{O}(s)\right\|=\left\|\bar{T}_{O}(s)\right\|$.

The following result gives an analytic constraint that must be satisfied by the output complementary sensitivity function of any stable SITO feedback system whose plant direction changes with frequency [15].

Proposition 4.1 Assume that the SITO feedback system is stable. Then

$$
\begin{aligned}
\frac{2}{\pi} \int_{0}^{\infty} \log \left(\frac{\left\|\tilde{T}_{O \cdot 1}(j \omega)\right\|}{\left|T_{r}(0)\right|}\right) \frac{d \omega}{\omega^{2}} \geq & 2\left[\sum_{i=1}^{N_{\alpha}} \frac{1}{\alpha_{i}}+\sum_{i=1}^{N_{\rho}} \frac{1}{\rho_{i}}\right] \\
& +\operatorname{Var}(P)-\tilde{K}_{v 11}^{-1},
\end{aligned}
$$

where $\alpha_{i}$ and $\rho_{i}$ are defined in (3.5), $\operatorname{Var}(P)$ is given in (3.7), and $\bar{K}_{v 11}^{-1}$ is defined by

$$
\bar{K}_{v 11}^{-1} \triangleq-\lim _{s \rightarrow 0} \frac{d}{d s} \frac{\bar{T}_{O 11}(s)}{\tilde{T}_{O 11}(0)} .
$$

The term $\tilde{K}_{v 11}^{-1}$ is related to the transient response of the closed loop system, see [15] for more discussion.

Clearly the terms containing $\alpha_{i}, \rho_{i}$ and $\operatorname{Var}(P)$ in the above integral constraint provide a link with the cheap control tracking cost.

Lemma 4.2 Assume that the feedback system is stable. Then

$$
\frac{2}{\pi} \int_{0}^{\infty} \log \left(\frac{\left\|\tilde{T}_{O \cdot 1}(j \omega)\right\|}{\left|T_{I}(0)\right|}\right) \frac{d \omega}{\omega^{2}} \geq \lim _{\varepsilon \rightarrow 0} J^{o p t}(r, \varepsilon)-\tilde{K}_{v 11}^{-1}
$$

We see from (4.1) that the cheap control tracking cost appears in an integral formula which applies to all single degree of freedom linear time invariant stabilizing controllers for SITO systems. A parallel link exists for single input single output (SISO) systems, provided by the term $\sum_{i=1}^{N_{\alpha}} 2 / \alpha_{i}$, where $\alpha_{i}$ are the non-minimum phase zeros of the scalar system [11]. As expected, in the STTO case the linking term also contains information about the variation with frequency of the plant direction. In the case where the plant direction is constant with frequency the results coincide with the SISO case.

\section{Example}

Example 5.1 Consider the plant

$$
P(s)=N(s) K(s)=\left[\begin{array}{l}
(1+\zeta) s+1 \\
(1-\zeta) s+1
\end{array}\right] \frac{1}{(s+2)^{2}},
$$

which, in DC coordinates takes the form

$$
\tilde{P}(s)=\tilde{N}(s) K(s)=\sqrt{2}\left[\begin{array}{c}
s+1 \\
\zeta s
\end{array}\right] \frac{1}{(s+2)^{2}} .
$$

The polynomial $\Delta=N^{\mathbf{T}} N$ has only one ORHP zero, $\delta_{1}=\frac{1}{\sqrt{1+\zeta^{2}}}$, and the $(1,1)$-element of $\bar{N}$ has a zero at $-\lambda_{1}=-1$ (cf. (3.5)). Using Theorem 3.2 it follows that

$$
\lim _{\varepsilon \rightarrow 0} J^{o p t}(r, \varepsilon)=\sqrt{1+\zeta^{2}}-1
$$

Note that (5.1) has no non-minimum phase zeros; the cheap control cost is entirely due to the change in the direction of the plant with frequency. If $\zeta \gg 1$, then the plant direction, given as the range of $N(s)$ in (5.1), changes a lot with frequency. This directly translates into a large value for the cheap control tracking cost. On the other hand, $\zeta \ll 1$ corresponds to little variation with frequency of the plant direction and, hence, to a small value for the cheap control tracking cost.

Example 5.2 Consider the control of a cart of mass $M$ attached to a ball of mass $m$ with a massless rod of length $l$. For a detailed discussion of this system see Doyle, Francis and Tannenbaum [1]. The mass $M$ slides in the direction $x$ on a horizontal surface; the angle that the rod makes with the vertical is denoted by $\theta$. The input to the system is a horizontal force $u$ on the cart. Denote by $g$ the acceleration due to gravity. Linearising about the stable equilibrium when the ball is directly below the cart yields the following transfer function:

$$
\left[\begin{array}{l}
X(s) \\
\Theta(s)
\end{array}\right]=\frac{1}{s^{2}\left(M l s^{2}+(M+m) g\right)}\left[\begin{array}{c}
l s^{2}+g \\
s^{2}
\end{array}\right] U(s) .
$$

The plant is already in DC coordinates, and has no NMP zeros. Consider the case of a unit step change in the reference signal. Figure 1 shows a plot of the optimal cost, which was calculated using Theorem 3.2, for various pendulum lengths $l$. This shows that the optimal cost increases for longer pendulum lengths. If the cost $\lim _{\varepsilon \rightarrow 0} J^{o p t}$ becomes large, then it is necessary to have a longer settling time, otherwise large transient signals will occur. Therefore, as the longer the length of the pendulum, the longer the settling time of the closed loop system.

\section{Conclusion}

This paper has extended the results of Qiu and Davison [8] concerning the cheap control of square linear systems to linear systems that are not right invertible. 


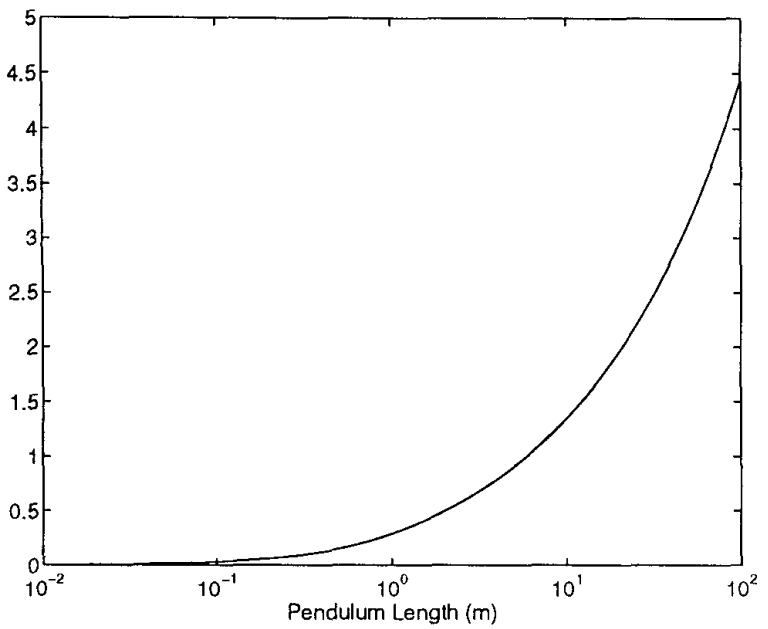

Figure 1: Cheap control cost vs. Pendulum length

If the plant is square, then $\lim _{\varepsilon \rightarrow 0} J^{o p t}(r, \varepsilon)=r_{1}^{\top} H r_{1}$, and trace $H$ is dependent only on the NMP zeros of the plant.

For the case in which the plant is not right invertible, we have provided a simple formula for trace $H$. In this case, trace $H$ depends not only on the NMP zeros of the plant, but also on the variation of the plant direction with frequency.

Previous work has shown a link between the cheap control results of Qiu and Davison and integral relations which constrain the output complementary sensitivity function [11]. Our results show that if the plant is not right invertible, then there is an analogous linkage between integral relations developed for SITO plants and the cheap control cost.

\section{References}

[1] John C. Doyle, Bruce A. Francis, and Allen R. Tannenbaum. Feedback Control Theory. Macmillan, 1992.

[2] Abbas Emami-Naeini and Steve M. Rock. On asymptotic behaviour of non-square linear optimal regulators. In Proceedings of the 23rd Conference on Decision and Control, volume 3, pages 1762-1763, Las Vegas, December 1984.

[3] Bruce A. Francis. The optimal linear-quadratic time-invariant regulator with cheap control. IEEE Transactions on Automatic Control, AC-24(4):616621, August 1979.

[4] J. S. Freudenberg and R. H. Middleton. Properties of single input, two output feedback systems. Submitted to the International Journal of Control, 1998.

[5] M. J. Grimble and M. J. Johnson. Optimal Con- trol and Stochastic Estimation Theory and Applications, volume 1. John Wiley, New York, 1988.

[6] Thomas Kailath. Linear Systems. Prentice-Hall, 1980.

[7] H. Kwakernaak and R. Sivan. The maximally ahcievable accuracy of linear optimal regulators and linear optimal filters. IEEE Transactions on Automatic Control, 17(1):79-86, 1972.

[8] L. Qiu and E. J. Davison. Performance limitations of non-minimum phase systems in the servomechanism problem. Automatica, 29(2):337-349, 1993.

[9] A. Saberi and P. Sannuti. Cheap and singular controls for linear quadratic regulators. IEEE Transactions on Automatic Control, AC-32(3):208-219, March 1987.

[10] M. M. Seron, J. H. Braslavsky, and G. C. Goodwin. Fundamental Limitations in Filtering and Control. Springer, 1997.

[11] M. M. Seron, J. H. Braslavsky, P. V. Kokotović, and D. Q. Mayne. Feedback limitations in nonlinear systems: From bode integrals to cheap control. Technical report, University of California, Santa Barbera, 1997.

[12] U. Shaked. Singular and cheap optimal control: the minimum and nonminimum phase cases. Technical Report TWISK 181, National Research Institute for Mathematical Sciences, Pretoria, Republic of South Africa, 1980.

[13] J. G. Truxal. Automatic Feedback Control System Synthesis. Mc-Graw Hill, 1955.

[14] A. R. Woodyatt, J. S. Freudenberg, and R. H. Middleton. A design tradeoff for single input two output feedback systems. In Proceedings of the 35th Allerton Conference on Communication, Control and Computing, October 1997.

[15] A. R. Woodyatt, J. S. Freudenberg, and R. H. Middleton. Integral constraints for single input two output feedback systems. Technical report, University of Newcastle, 1997. EE9703.

[16] A.R. Woodyatt, J.S. Freudenberg, M.M. Seron, and R. H. Middleton. Cheap control tracking performance for non-right-invertible systems. Technical Report EE9818, University of Newcastle, 1998.

[17] Kemin Zhou, John C. Doyle, and Keith Glover. Robust and Optimal Control. Prentice Hall, 1996. 